\title{
QUALIDADE DE VIDA DO PACIENTE COM CÂNCER COLORRETAL EM QUIMIOTERAPIA AMBULAT ORIALa
}

\author{
Patrícia Lemos CHAVE S ${ }^{b}$ M aria I sabel Pinto Coelho G OR INIc
}

\section{RESUM 0}

T rata-se de um estudo transversal com abordagem quantitativa descritiva, no qual buscou-se avaliar a qualidade de vida (QV) do paciente com câncer color retal em quimioterapia ambulatorial. A pesquisa foi desenvolvida em uma U nidade de Quimioterapia A mbulatorial em um hospital do sul do B rasil, cujos pacientes eram portadores do diagnóstico de câncer colorretal e foram submetidos ao tratamento com o Protocolo $5 \mathrm{~F} \mathrm{U}$. A amostra contou com 48 participantes que estavam em tratamento quimioterápico por um período de seis meses. U tilizou-se como instrumento um questionário, O W H OQOL - B ref. N os resultados encontrados, prevaleceu a idade de 50 anos ou mais e possuíam no mínimo um mês e no máximo 11 meses de tratamento. Os domínios do W H OQOL -Bref afetados mais significativamente for am o psicológico e o das relações sociais, respectivamente, havendo diferença nas respostas quanto à QV geral naqueles que estavam no $1^{0}$ ciclo detratamento daquel es que já se encontravam no 60 ciclo.

D escritores: Qualidade de vida. Quimioter apia. Enfermagem oncológica.

\section{RESUMEN}

E ste es un estudio transversal con un enfoque cuantitativo descriptivo, que buscó evaluar la calidad de vida (Q OL ) de los pacientes con cáncer colorrectal en quimioterapia ambulatorial. La investigación fue desar rollada en una unidad de quimioterapia O utpatient Clinic en un hospital del sur de B rasil, cuyos pacientes tenían el diagnóstico de cáncer col or rectal y que se presentaron al tratamiento con el P rotocolo $5 \mathrm{~F} \mathrm{U.} \mathrm{L} \mathrm{a} \mathrm{muestra} \mathrm{ha} \mathrm{contado} \mathrm{con} 48$ participantes que se encontraban en quimioterapia por un período de 6 meses. Se utilizó como instrumento un cuestionario, W H O Q O L - B ref. E n los resultados, prevaleció la edad de 50 años o más y tenían al menos un mes y un máximo de 11 meses de tratamiento. L as áreas de W H OQ O L - B ref afectadas más significativamente eran las esferas psic cológica y las relaciones sociales, respecti vamente, con diferencia en las respuestas de Q $0 \mathrm{~L}$ general las que estaban en 1 ciclo de aquel los que ya estaban en el ciclo 6T H .

Descriptores: Calidad de vida. Quimioterapia. E nfermería oncológica.

Título: Calidad de vida de los pacientes con cáncer color rectal en quimioterapia ambulatoria.

\section{ABST RACT}

This is a cross-sectional study with a descriptive quantitative approach, which aimed to assess the quality of life (QOL ) of patients with colorectal cancer receiving outpatient chemotherapy. T he research was conducted in an 0 utpatient Chemotherapy U nit at a hospital in southern B razil, whose patients were diagnosed with colorectal cancer and were treated with the 5- $\mathrm{F} U$ protocol. Thesample had 48 participants who wereundergoing chemotherapy for a period of six months. A questionnaire, the WHOQOL-B ref, was used as an instrument. In the results, the age of 50 years or more with at least a month and a maximum of 11 months of treatment prevailed. Thedomains of theW HOQOL - B ref more significantly affected were the psychological and the social relations one, respectively, with significant differences in responses regarding overall QOL in those who w ere in the first cycle of treatment from those al ready in the 6th cycle.

D escriptors: Quality of life. D rug therapy. Oncologic nursing.

T itle: Quality of life of patients with colorectal cancer receiving outpatient chemotherapy.

\footnotetext{
a Artigo originado da dissertação de M estrado apresentada em 2010 ao Programa de Pós-Graduação em Enfermagem da U niversidade Federal do Rio Grande do Sul (UFRGS).

b M estre em E nfermagem, Enfermeira da E ducação Permanente no H ospital São L ucas da Pontifícia U niversidade Católica do Rio G rande do Sul (PU CRS), Porto Alegre, Rio G rande do Sul, Brasil.

c D outora em E ducação, Professora A djunta da Escola de Enfermagem da UFRGS, Porto Alegre, Rio Grande do Sul, Brasil.
} 


\section{INT RODUÇÃO}

0 câncer de cólon e reto configura-se como a terceira causa mais comum de câncer no mundo em ambos os sexos e a segunda causa em países desenvolvidos ${ }^{(1)}$.

0 vivenciar um câncer pode desencadear reflexões sobre a vida, pois, uma vez instalada a doença, a pessoa necessita de uma série de mudanças nos hábitos de vida e entre elas um acompanhamento rigoroso de seu estado de saúde, afinal, em alguns casos, as recidivas da doença são inevitáveis. A descoberta deste diagnóstico ocorre dentro de um contexto familiar, desencadeando mudanças na família como um todo, de forma que os familiares, em maior ou menor grau, são afetados pelas situações decorrentes da doença ${ }^{(2)}$.

Visando a diminuição do impacto das possíveis alterações físicas e emocionais e, também, dos efeitos colater ais adversos do tratamento quimioterápico e, numa tentativa de proporcionar uma maior qualidade de vida aos pacientes, a oncologia surge como uma especialidade que, por excelência, se viu confrontada com a necessidade de avaliar as condições de vida dos pacientes que tinham sua sobrevida aumentada com os tratamentos propostos, já que muitas vezes na busca de acrescentar "anos à vida" era deixado de lado a necessidade de acrescentar "vida aos anos"(3).

N esta perspectiva, a mensuração da Qualidade de Vida (QV) é um importante recurso para avaliar os resultados do tratamento na perspectiva do paciente; sendo fundamental que os trabal hadores da enfermagem estejam instrumentalizados para realizar a avaliação dos pacientes, monitorando as manifestações clínicas, os efeitos colaterais adversos e as repercussões do tratamento quimioterápico, visando à diminuição do impacto das possíveis alter ações.

E studar a qualidade de vida é fundamental para a presença de intervenção que promovam bemestar e/ ou o estar-bem destes pacientes, cujo prognóstico nem sempre é o mel hor.

Dessa forma, o presente estudo tem como questão norteadora: Como o paciente com câncer colorretal em quimioterapia ambulatorial avalia sua qualidade de vida? E teve como objetivos avaliar a qual idade de vida dos pacientes com câncer colorretal em quimioterapia ambulatorial utilizando como instrumento $\mathrm{O}$ World $\mathrm{H}$ ealth Organization's Quality of Life Bref (W HOQOL -
B ref) ${ }^{d}$, bem como descrever as características dos pacientes com câncer colorretal em quimioterapia ambulatorial.

\section{TRAJET ÓRIA METODOLÓGICA}

T rata-se de um estudo transversal com abordagem quantitativa descritiva, desenvolvido no H ospital de Clínicas de Porto Alegre (HCPA), na U nidade de Quimioterapia A mbulatorial (UQA) com pacientes portadores do diagnóstico de câncer colorretal submetidos ao tratamento com o Protocolo 5FU (Fluorouracil + ácido folínico). Este protocolo é uma combinação de drogas utilizada para o tratamento do câncer colorretal (4).

Os sujeitos da pesquisa foram pacientes adultos em acompanhamento ambulatorial portadores de câncer colorretal submetidos ao tratamento quimioterápico adjuvante com o protocolo $5 \mathrm{FU}$. Foram excluídos os pacientes portadores de patologias crônicas descompensadas, pacientes com recidiva e pacientes em fase terminal.

A amostra do estudo foi intencional e teve no total 48 participantes que, no período da coleta, estavam fazendo o tratamento com o Protocolo 5FU e atenderam aos critérios de inclusão da pesquisa: adultos, com idade superior a 18 anos; submetidos ao tratamento quimioterápico adjuvante com o protocolo 5F U; lúcidos, orientados e comunicativos; com disponibilidade e interesse em participar da pesquisa; conveniados pel o Sistema Ú nico de Saúde (SU S); que iniciaram o P rotocolo $5 \mathrm{FU}$ no período de 10 de julho de 2009 a 31 de julho de 2009; a partir do 1 - ciclo do protocolo. Foram excluídos se te participantes por não atenderem a pelo menos um dos critérios de inclusão.

A amostra foi estabelecida considerando nível de significância de 0,05 . A estimativa do tamanho da amostra foi de 50 participantes, prevendo $5 \%$ de perdas por não atenderem aos critérios de inclusão da pesquisa.

A coleta de dados foi realizada no período de seis meses e ocorreu a partir do 10 ciclo de tratamento, buscando atender aos objetivos propostos, utilizando o W orld $\mathrm{H}$ ealth Organization's Quality of L ife Bref (WHOQOL - B ref): um questionário que avalia a qualidade de vida, sendo este, já validado e traduzido para o português(4).

\footnotetext{
¿ Questionário que avalia qualidade de vida dentro de uma perspectiva genuinamente internacional, desenvolvido pela Organização M undial da Saúde(4).
} 
O WHOQOL - Bref é um instrumento de 26 questões sobre qualidade de vida, dividido em quatro domínios: físico, psicológico, relações sociais e meio-ambiente, com duas questões gerais sobre QV ${ }^{(4)}$. As duas questões ver sam sobre como 0 indivíduo avalia sua sua QV e sobre quão satisfeito está com sua saúde.

T ambém foram coletados dados que caracterizassem a amostra, tais como: idade, sexo, estadiamento do tumor, tempo de tratamento, ciclo do tratamento do Protocolo 5F U e a presença ou não de colostomia e se é transitória ou per manente.

Cada participante foi abordado apenas uma vez, respondendo ao questionário e infor mando 0 ciclo de tratamento em que estava no período da coleta; e buscou-se pacientes que estavam nos diferentes ciclos de tratamento do Protocolo 5FU para responder ao instrumento.

Os dados foram organizados em forma de planilha do Programa Excel for W indows e após foram analisados pelo software Statistical Package for the Social Sciences (SPSS), a análise dos dados foi gerenciada por meio do Programa SPSS 18.0, junto ao serviço de Bioestatística oferecido pelo G rupo de Pesquisa da Pós-G raduação (G PPG ) do HCPA.

Foi solicitado uma autorização por escrito à chefia de enfermagem da instituição hospitalar envolvida e aprovado pelo Comitê de É tica (parecer no 09-224/2009). Além disso, em atenção à $R e-$ solução 196/ 96 do Consel ho Nacional de Saúde(5), foi solicitado o Termo de Consentimento Livre e Esclarecido (T CLE) do paciente, informando o objetivo e metodologia do trabal ho, assegurando 0 anonimato de suas repostas, assim como o seu direito de não participarem do estudo.

\section{RESULTADOS E DISCUSSÃO}

Os resultados foram obtidos através de quatro análises distintas: estatística descritiva, mediante as médias das variáveis independentes (ou fatores do estudo), de modo a verificar as características dos pacientes com câncer colorretal em quimioterapia ambulatorial; 0 teste t de Student, para comparar as variáveis de interesse; análise de variância, para comparar as variáveis em que estavam envolvidos três ou mais grupos (variáveis independentes e dependentes), ou seja, para comparar os domínios do instrumento com os fatores do estudo; e o teste de comparações múltiplas de T ukey que permitiu estabelecer a diferença mínima significante, entre as médias das variáveis dependentes. A T abela 1 apresenta características da amostra estudada.

T abela 1 - Caracterização dos pacientes com câncer colorretal $(\mathrm{N}=48)$. Porto Alegre, RS, 20092010.

\begin{tabular}{lrr}
\hline Características sociais & $\mathbf{n}$ & $\%$ \\
\hline Idade & & \\
$30-39$ & 5 & 10,4 \\
$40-49$ & 29 & 29,2 \\
$\geq 50$ & 24 & 50,4 \\
Sexo & 24 & 50,0 \\
$\quad$ Feminino & & \\
M asculino & & \\
Ciclo do tratamento do & 4 & 8,3 \\
protocolo 5F U & 11 & 22,9 \\
1 & 9 & 18,8 \\
2 & 8 & 16,7 \\
3 & 8 & 16,7 \\
4 & 8 & 16,7 \\
5 & & \\
6 & 40 & 83,3 \\
Presença de colostomia & 8 & 16,7 \\
N & - & - \\
S - T & & \\
S - P & & \\
\hline
\end{tabular}

Legenda: $\mathrm{N}=\mathrm{N}$ ão; $\mathrm{S}=\mathrm{Sim} ; \mathrm{T}=\mathrm{T}$ emporária; $\mathrm{P}=$ Permanente.

No que diz respeito ao estadiamento do tumor, não foi evidenciado na Tabela 1, pois todos, ou seja, 100\% dos participantes desse estudo estavam com Dukes $B$.

Quanto à idade $60,4 \%$ tem 50 anos ou mais, seguido de pacientes com idade entre 40 e 49 anos $(29,2 \%)$ e uma pequena quantidade, mas não menos importante de pacientes com idade entre $30 \mathrm{e}$ 39 anos (10,4\%), o que vai ao encontro dos dados da literatura.

Esses tumores são mais comuns em pessoas com mais de 50 anos, as quais devem se submeter anualmente a exames, já que entre os fatores que aumentam o risco de desenvolvimento da doença está a idade acima de 50 anos ${ }^{(1)}$.

No que diz respeito à presença ou não de coIostomia, a maioria dos pacientes em tratamento quimioterápico ambulatorial não apresentava coIostomia (83,3\%), o restante apresentava colostomia transitória $(16,7 \%)$ e nenhum apresentava colostomia permanente. 
A colostomia é a criação cirúrgica de uma abertura (ostoma) para dentro do cólon para eliminação de fezes. Pode ser utilizada quando o paciente apresenta qualquer problema que 0 impede de evacuar normalmente pelo ânus; podendo ser temporários ou per manentes ${ }^{(6)}$. No caso dos ostomas intestinais, geralmente, são realizados para evitar que as fezes passem pelo local operado antes da cicatrização completa; e são ditadas pela localização do tumor e extensão da invasão para dentro dos tecidos vizinhos.

$\mathrm{N} \mathrm{a}$ cirurgia para tratamento do câncer de reto, os ostomas temporários são fechados depois da cicatrização da cirurgia, o que ocorre geralmente após um mês da cirurgia. A pós o fechamento do ostoma, o paciente volta a evacuar pelo ânus.

Já, em relação ao ciclo de tratamento do Protocolo 5FU em que estavam a amostra foi bastante linear, demonstrando pouca diferença de pacientes em cada ciclo: $1^{\circ}$ ciclo (8,3\%), $2^{\circ}$ ciclo ( $\left.22,9 \%\right)$, $3^{\circ}$ ciclo $(18,8 \%)$ e 4우 5 o e 60 ciclos $(16,7 \%)$ cada um. E ste dado sugere que a maioria dos pacientes que estão em acompanhamento ambulatorial para tratamento com o Protocolo $5 \mathrm{~F} \mathrm{U}$ conseguem dar uma continuidade ao mesmo até o fim do tratamento.

0 objetivo de um estadiamento no paciente com câncer é o de identificar não somente a extensão locorregional da lesão primária, mas também a sua extensão a distância. A mensuração do antígeno carcinoembrionário (CE A) tem grande importância para o prognóstico da doença ${ }^{(7)}$.

A quimioterapia adjuvante em pacientes com D ukes $B$ de câncer de cólon é eficaz, embora o efeito sobre a sobrevivência é pequena, no entanto, a quimioterapia adjuvante reduziu o risco relativo de recidiva do tumor ${ }^{(8)}$.
No que se refere ao tempo de tratamento dos pacientes com Protocolo 5F U a média foi de 5,56 meses de tratamento. Sendo o mínimo de tempo encontrado de um mês o que configura que o paciente está no início do tratamento, e o máximo de 11 meses, demonstrando que nem sempre é possível concluir o tratamento no tempo previsto.

Pacientes com Dukes B apresentam indicação de tratamento adjuvante com quimioterapia à base de $5 \mathrm{FU}$, cujo esquema recomendado é o $5 \mathrm{~F} \mathrm{U}$ + ácido folínico durante seis meses; sendo administrado durante cinco dias consecutivos, com interval os de 21 dias, totalizando seis ciclos do tratamento ${ }^{(9)}$.

Entretanto, é comum durante o tratamento quimioterápico, o paciente vir a apresentar efeitos colaterais a droga recebida; especial mente com 0 Protocolo 5FU a toxidade gastrintestinal é a mais presente.

A quimioterapia é um tratamento sistêmico que tem um grande impacto sobre a divisão das células tumorais, provoca toxicidade pelo efeito deletério sobre a divisão das células normais do corpo tais como, a medula óssea ou trato gastrointestinal $^{(10)}$.

Entre eles, os mais comuns associados à quimioterapia sistêmica, são as náuseas e vômitos, a mucosite e a diarréia; que podem afetar a condição nutricional, o equilíbrio hidroeletrolítico e a qualidade de vida do paciente. Além disso, fatores hematológicos podem aparecer (toxidade hematológica), tais como a anemia, leucopenia, neutropenia(10); fatores esses que podem vir a interromper por um período o tratamento com o Protocolo $5 \mathrm{FU}$, fazendo com que este dure um pouco mais do que tempo previsto inicialmente, que era de 6 meses.

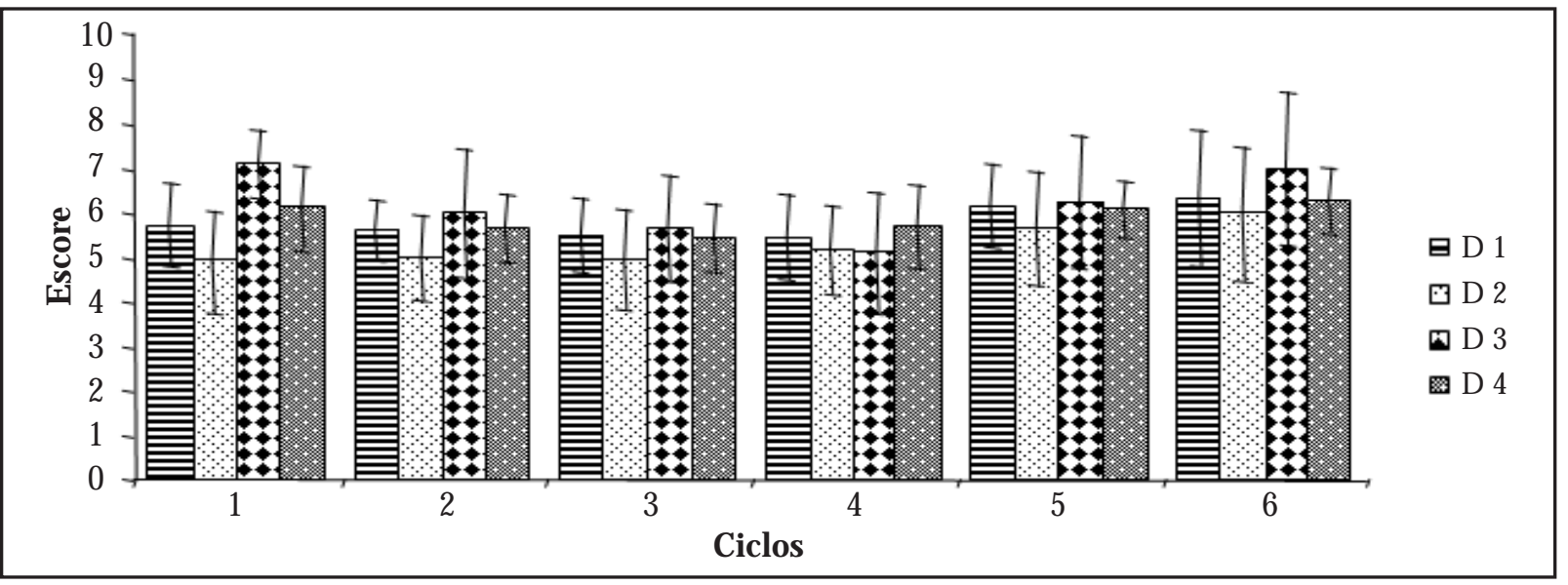

G ráfico 1 - Domínios do W H OQOL - B ref. Porto Alegre, RS, 2009-2010. 
No G ráfico 1, a seguir, foi feito um cruzamento dos dados nos seis ciclos de tratamento com 0 Protocolo 5FU com os domínios do W HOQOL Bref.

O G ráfico 1 demonstra uma linearidade entre os dados e os respectivos domínios do W $\mathrm{H} \mathrm{O}$ Q O L - B ref, o que sugere uma qual idade de vida boa, levando-se em consider ação que todos el es se mantiveram num padrão de no mínimo 50\%.

A seguir são apresentados os quatro domínios, os respectivos subdomínios e o número da questão a que correspondem:

a) domínio 1 - físico: dor e desconforto; energia e fadiga; sono e repouso; mobilidade; atividades da vida cotidiana; dependência de medicações ou tratamentos; capacidade de trabal ho;

b) domínio 2 - psicológico: sentimentos positivos; pensar, aprender, memória e concentração; auto-estima; imagem corporal a aparência; sentimentos negativos; espiritualidade/ religião/ crenças pessoais;

c) domínio 3 - relações sociais: relações pessoais; suporte social; atividade sexual;

d) domínio 4 - meio ambiente: segurança física e proteção; ambiente no lar; recur sos financeiros; cuidados de saúde e sociais: disponibilidade e qualidade; oportunidades de adquirir novas informações e habilidades; participação e oportunidades de recreação / lazer; ambiente físico: poluição, ruído, clima, trânsito; transporte.

A T abela 2 mostra os valores obtidos nos domínios e Qualidade deVida (QV) geral do W H OQOL - B ref.

T abela 2 - Valores obtidos nos domínios e Q ualidade de Vida (QV) geral do W H O Q O L - B ref. Porto Alegre, RS, 2009-2010.

\begin{tabular}{lccc}
\hline $\begin{array}{l}\text { D omínios e QV } \\
\text { geral (Q1 e Q2) }\end{array}$ & Média & M ediana & $\begin{array}{c}\text { D esvio } \\
\text { padrão }\end{array}$ \\
\hline 1 - Físico & 13,25 & 13,14 & 1,91 \\
2 - Psicológico & 12,44 & 12,00 & 2,29 \\
3 - Relações Sociais & 13,72 & 14,00 & 2,91 \\
4 - M eio A mbiente & 13,32 & 13,25 & 1,55 \\
Q1 & 14,58 & 12,50 & 5,36 \\
Q2 & 11,45 & 12,50 & 7,10 \\
\hline
\end{tabular}

Observou-se que no W H OQO L - B ref, o domínio com escore mais alto foi o de Relações Sociais (domínio 3), com média de 13,72 e o desvio padrão de 1,55 . Por outro lado o domínio com escore mais baixo foi o Psicológico (domínio 2) com média de 12,44 e desvio padrão de 2,29.

A T abela 2 mostra que não houve diferença significativa nas respostas dos participantes entre os domínios, e tampouco quanto a suas percepções de qualidade de vida.

E m relação a QV geral, Q 1 e Q 2 representam como o paciente aval ia sua qual idade de vida e quão satisfeito está com sua saúde, respectivamente. Foi obser vado que os participantes aval iam sua qual idade de vida de for ma positiva bem como, demonstraram também, estar satisfeitos quanto a sua saúde; percebido nos escores Q $1(14,58)$ e Q $2(11,45)$. $\mathrm{N}$ esse sentido, pode-se inferir que os participantes deste estudo avaliam positivamente sua qualidade de vida, mas não estão satisfeitos com sua saúde. E ste achado pode ser atribuído a situação a qual os participantes desta pesquisa estão vivenciando no momento.

O se descobrir com câncer e/ ou viver o tratamento de uma doença considerada, ainda por muitos, como incurável, pode acarretar em uma série de angústias, dúvidas, expectativas, crenças e fé abaladas ${ }^{(11)}$. E videnciado no g ráfico I, cujo domínio P sicológico foi o que apresentou porcentagens menores em relação aos outros domínios, o que justificaria nossa hipótese.

E m contrapartida, o domínio das Relações sociais se mostrou com as médias mais el evadas, já constatado na T abela 2 e visual izado no $\mathrm{G}$ ráfico 1. 0 que pode ser atribuído as questões de apoio da família e amigos no seu momento atual.

N este estudo, homens e mulheres tiveram uma percepção semel hante de qualidade de vida, ou seja, não houve diferença significativa quanto a sua avaliação de QV.

A média entre os sexos, relacionada a cada domínio do W H OQOL - B ref, mostrou-selinear entre as respostas no instrumento. 0 que também pode ser justificado em função da amostra total ter sido igual o número de pacientes de cada sexo.

No que diz respeito à presença ou não de colostomia, o domínio 2 (psicológico) se mostrou mais significativo em relação as demais. A queles que tinham colostomia, mesmo que transitória tiveram respostas diferenciadas, ou seja, respostas mais negativas que aqueles que não a tinham. Foi onde os sentimentos quanto a sua auto-estima associada a imagem corporal e aparência, sentimentos positivos e/ ou negativos estiveram mais afetados. 
As alter ações da integ ridade físico-emocional como desconforto, dor, desfiguração, dependência e perda de auto-estima são relatadas pel os pacientes que percebem a qualidade de suas vidas profundamente alteradas em curto espaço de tempo ${ }^{(12)}$.

A auto-estima é um outro fator bastante importante, no câncer colorretal, al ém do estigma do câncer e de sua local ização corporal, o indivíduo tem suas preocupações ag ravadas pelas possibilidades terapêuticas, dentre as quais, o desvio do trânsito intestinal mediante a realização de um estoma. A pessoa ostomizada, além de sobreviver ao câncer, passa a assumir outras incumbências em presença de tal derivação(11,13).

E m contrapartida, no domínio 3 (relações sociais), aquel es que não têm presença de colostomia se mostraram mais positivos nas respostas do que o que tinham a colostomia. 0 que demonstra, claramente, a influência de sua condição física e psicológica nas suas relações pessoais, bem como na sua percepção de qualidade de vida.

A T abela 3 apresenta a média em cada ciclo em relação as duas questões gerais sobre QV, ou seja, aquelas que versam sobre como o indivíduo avalia sua QV e sobre o quão satisfeito está com sua saúde.

T abela 3 - M édia da Qualidade de Vida (QV) geral em cada ciclo do tratamento com Protocolo 5F U. Porto A legre, RS, 2009-2010.

\begin{tabular}{lrcc}
\hline \multirow{2}{*}{ Ciclo } & \multirow{2}{*}{} & \multicolumn{2}{c}{ Q 1 e Q 2 } \\
\cline { 3 - 4 } & & $\mathbf{1}$ & $\mathbf{2}$ \\
\hline 1 & 4 & 9,5000 & - \\
2 & 11 & 10,7273 & 10,7273 \\
3 & 8 & 11,7500 & 11,7500 \\
4 & 9 & 11,7778 & 11,7778 \\
5 & 8 & 14,2500 & 14,2500 \\
6 & 8 & - & 15,2500 \\
Sig. & - & .111 & .145 \\
\hline
\end{tabular}

Legenda: Sig.: nível de significância (probabilidade de erro). Nota: $p \geq 0.05$.

$\mathrm{Na}$ Tabela 3 foi utilizado o teste de comparações múltiplas de T ukey para que fosse observado a diferença mínima significante, ou seja, a menor difer ença entre as médias da amostra.

A Tabela 3 mostra que, de uma maneira geral, os pacientes consideram que tem boa qualidade de vida. No entanto, houve difer ença significativa entre o 1 o e o 60 ciclo de tratamento com 0
Protocolo 5FU, demonstrando divergência na percepção daquel es que estão iniciando o tratamento com aqueles que estão na sua última etapa.

E videncia-se também que houve uma crescente no que diz respeito a sua satisfação e avaliação de sua QV, percebe-se que a medida que vão evo-luindo nos ciclos de tratamento com o protocolo 5F U, ou seja, na medida em que se aproxima o término do tratamento, vão relatando de forma mais positiva sua QV.

Viver com uma doença como o câncer colorretal é um desafio. Além de enfrentar a própria doença, o paciente enfrenta uma série de preocupações, sentimentos e expectativas que podem tornar a sua vida ainda mais difícil. 0 paciente com câncer colorretal, assim como qualquer outro paciente com câncer, pode precisar de ajuda tanto nos aspectos emocionais quanto práticos de sua doença(11)

I sto é visualizado na tabela acima, onde aqueles pacientes que a recém se descobriram com câncer e/ ou iniciaram o tratamento com câncer se mostraram mais negativos quanto a sua qualidade de vida, do que aqueles que já estão no final do tratamento.

De acordo com dados nacionais de estimativa do câncer, a sobrevida para esse tipo de neoplasia pode ser considerada boa, relacionando-se principal mente a um diagnóstico precoce, assim como os achados referentes aos pacientes investigados nesta pesquisa(1).

\section{CONSIDERAÇÕES FINAIS}

Há alguns anos, a preocupação da equipe de saúde em relação ao câncer era a sobrevivência dos pacientes. $\mathrm{H}$ oje, o foco do tratamento mudou, isto é, a preocupação inclui a qualidade de vida que o paciente com câncer vai ter durante e após o tratamento oncológico.

A qualidade de vida é uma importante medida de resultados, pois permite uma maior atenção a percepção dos indivíduos sobre seu modo de conduzir sua vida. Por essa razão, as avaliações foram feitas a partir do $1^{0}$ ciclo de tratamento com o protocolo $5 \mathrm{FU}$ e foram excluídos aqueles que já tinham experiência prévia com o tratamento, para que não houvesse nenhum viés de resposta nesse sentido.

0 conjunto dos resultados apresentados permitiu visualizar que os pacientes com câncer co- 
Iorretal em quimioterapia ambulatorial avaliam de forma positiva sua qualidade de vida.

E sta pesquisa avaliou a qualidade de vida de pacientes com câncer colorretal em quimioterapia ambulatorial. Além disso, vimos que a qualidade de vida é resultado da combinação de fatores subjetivos (como o grau de satisfação geral de um indivíduo com a própria vida) e de fatores objetivos, como o bem-estar material, boas relações familiares, disposição para tratamento do câncer, entre outros fatores, enfim, vários itens que somados proporcionam tranqüilidade, confiança, segurança, bem-estar. A qualidade de vida precisa suprir as necessidades humanas integ rais, em seus aspectos físicos, psicológicos, sociais e espirituais.

A nalisar a QV dos pacientes com câncer color retal é um importante caminho para se conhecer melhor aqueles a quem se presta cuidado, permitindo assim, que sejam criadas ou mesmo, proporcionadas medidas mais adequadas no cuidado a esse tipo de paciente.

U ma contribuição relevante refere-se a possibilidade de um estudo comparativo entre a avaliação da qualidade de vida feita pelo paciente e outra, de como é a percepção dos enfermeiros da qualidade de vida desse paciente. É importante considerar a percepção dos pacientes com câncer em quimioterapia ambulatorial frente à sua qualidade de vida, para então, elaborar a assistência de enfermagem em situações que requer em o controle dos efeitos adver sos e das conseqüências do tratamento.

U ma sugestão rel evante refere-se à condução de mais estudos nesta área e/ ou com esta temática, afim de buscar alternativas que subsidiem novas práticas de cuidado a esse paciente, oportunizandoos uma melhor qualidade de vida, bem como 0 de fornecer dados que ampliem o conhecimento dos profissionais atuantes nessa área.

\section{REFERÊNCIAS}

1 M inistério da Saúde (BR), Secretaria de Atenção à Saúde, Instituto N acional do Câncer, Coordenação de Prevenção e Vigilância de Câncer. E stimativas 2010: incidência de câncer no Brasil. Rio de Janeiro; 2010.
2 Salci M A, M arcon SS. A convivência com o fantasma do câncer. Rev G aúcha E nferm. 2010;31(1):18-25.

$3 \mathrm{~K}$ atscnig M . Instrumentos de avaliação de qualidade de vida [ I nter net] . Porto A leg re: Organização M undial de Saúde/ W orld H ealth O rganization Quality of Life Assessment; 1998 [ citado 2011 set 17] . Disponível em: http:// www.ufrgs.br/psiq/ whoqol.html.

4 F leck M PA. A avaliação de qualidade de vida: guia para profissionais de saúde. Porto Alegre: Artmed; 2008.

5 M inistério da Saúde (BR ), Consel ho N acional de Saúde. Resolução 196, de 10 de outubro de 1996: diretrizes e normas regulamentadoras de pesquisa envolvendo seres humanos. Brasília (D F ); 1996.

6 Smeltzer SE, Bare B. Brunner e Suddart: tratado de enfermagem médico-cirúrgica. 9a ed. Rio de Janeiro: Guanabara Koogan; 2006.

7 Cordeiro F. Diagnóstico, estadiamento e tratamento cirúrgico e multidisciplinar do câncer colorretal. São Paulo: A ssociação M édica Brasileira/ Consel ho F ederal de M edicina; 2001. (P rojeto Diretrizes).

8 Wolmark N, Rockette $\mathrm{H}, \mathrm{M}$ amounas EP. The relative efficacy of 5-FU + leucovorin (FU-LV), 5-FU + levamisole (FU-LEV) and 5-FU + leucovonn + levamisole (FU-LV-LEV) in patients with Dukes' B and $C$ carcinoma of the colon: first report of N SABP C-04. Proc Am Soc Clin Oncol. 1996;15:205.

9 Forones N M . G uia de medicina ambulatorial e hospitalar de oncologia. Barueri: M anole; 2005.

10 B onassa E M , Santana T R . E nfer magem em terapêutica oncológica. 3a ed. São Paulo: A theneu; 2006.

11 Chaves PL. Avaliação da qualidade de vida do paciente com câncer colorretal em quimioterapia ambulatorial [ dissertação]. Porto Alegre: Escola de Enfermagem, U niver sidade Federal do Rio G rande do Sul; 2010.

12 M ichelone APG, Santos VLCG. Qualidade de vida de adul tos com câncer colorretal com esem ostomia. Revista L atino-A m Enfermagem. 2004;12(6):875-83.

13 N icolussi AC, Sawada N O. Qualidade de vida de pacientes com câncer colorretal em terapia adjuvante. Acta Paul Enferm. 2009;22(2):349-63.

Recebido em: 24/ 11/ 2010

A provado em: 17/ 10/ 2011

\section{Author's address:}

Endereço da autora / Dirección del autor /

Patrícia L emos Chaves

Rua M iguel Couto, 601, ap. 12, M enino D eus

90850-050, Porto Alegre, RS

E-mail: pati80 chaves@ hotmail.com 